\title{
Prevalence of Resistance-Associated Mutations in Human Immunodeficiency Virus Type 1-Positive Individuals Failing HAART in Rio de Janeiro, Brazil
}

Rafael Brandão Varella, Selma Baía Ferreira, Márcia Braga de Castro, Marisa Dias Tavares and Mariano Gustavo Zalis Department of Infectious Diseases, Clementino Fraga Filho University Hospital, Federal University of Rio de Janeiro, Brazil

\begin{abstract}
We investigated the occurrence of HIV-1 antiretroviral resistance in individuals failing to respond to highly active antiretroviral therapy (HAART) attended by RENAGENO from 2001-2004. One hundred and seventeen patients were selected for this study; their plasma viral RNA was extracted and the PR and RT genes sequenced to examine subtype, genetic polymorphisms and mutations associated with resistance to antiretroviral drugs. HIV-1 sequence analysis showed that $86 / 100(86 \%)$ were infected with subtype $B, 7 /$ 100 (7\%) with subtype F and 7/100 (7\%) with RT/PR hybrid forms (2 D/B, 2 F/B, 2 B/F and 1 D/F). In 14 $(12 \%)$ of the samples, the subtype was not determined. The prevalence of resistance mutations was high (93.1\%), mainly in the RT gene. The most prevalent resistance mutations were: M184V (60.7\%), T215Y (49.6\%) and M41L (46.7\%) in the RT gene and L90M (19.6\%), M46I (16.2\%) and D30N (12.8\%) in the PR gene. The frequency of resistance mutations tended to increase from the first to the second therapeutic scheme failure $(p=0.079)$; but it stabilized after subsequent failures $(p=0.875)$. Our finding of a high frequency of drug resistant HIV-1 samples supports the need for continuous genotypic monitoring of patients failing HAART.
\end{abstract}

Key words: HIV-1, HAART, reverse transcriptase, protease, resistance.

Antiretroviral therapy (ARV) developed to control human immunodeficiency virus (HIV) infections includes nucleosidic and non-nucleosidic reverse transcriptase inhibitors (NRTIs and NNRTIs, respectively) and protease inhibitors (PIs) [1,2]. In order to improve clinical and virological responses, current therapy is composed of a triple-drug combination of at least two of these drug classes; this is known as highly active antiretroviral therapy (HAART) [3]. In Brazil, more than 130,000 people have free access to ARV [4]. This state policy has helped reduce AIDS incidence by $50 \%$ during recent years and has improved the quality of life of HIV-positive individuals in our country [5]. However, HIV resistance to ARV, leading to HAART failure, is inevitable in the course of infection [5], as is the transmission of resistant strains [6]. Resistance is explained by intense viral replication in the host $\left(10^{10}\right.$ particles per day) [7], associated with random mutations occurring in the viral genome, due to reverse transcriptase (RT)-polymerase errors $\left(10^{-4}\right.$ per incorporated nucleotide) $[8,9]$. The result of this combination is the emergence of different but related HIV genetic populations, known as quasispecies [10].

When mutations occur in certain sites of the protease (PR) and RT genes of the pol region of the HIV genome [11], which are responsible for vital enzymatic functions, resistance to ARV drugs is possible [12]. The consequence of HIVresistance is therapeutic failure, resulting in a plasmatic viral load boost and the appearance of opportunistic diseases $[13,14]$. Weak adhesion to treatment and late detection of viral

Received on 19 February 2008; revised 18 September 2008.

Address for correspondence: Dr. Rafael Brandão Varella. Laboratório de Infectologia e Parasitologia Molecular (LIPAM), Hospital Universitário Clementino Fraga Filho, Av. Brigaderio Trompowsky s/ n, Ilha do Fundão-Brazil. E-mail: rafael_varella@hotmail.com. resistance also reduce the odds of therapeutic regimen success and increases the transmission of resistant strains [15].

In order to evaluate the extension of resistance and distribution of subtypes in our country, the Brazilian government created the National Network for HIV Genotyping (RENAGENO) in 2001 [16], involving reference laboratories and a team of specialists in HIV resistance. Genotype testing has been found to be beneficial for guiding therapy in patients with HIV infection and virological failure [17-19]. We investigated the occurrence of HIV-1 resistance by analyzing RT and PR genes in individuals failing HAART.

\section{Material and Methods}

Patients

One hundred and seventeen patients selected for genotyping tests from 2001 to 2004 were attended by one of the RENAGENO-associated units, the Viral Load Laboratory of Federal University of Rio de Janeiro. Informed consent was obtained from each individual after the nature and possible consequences of the study had been fully explained. Selective criteria for genotyping tests were based on HIV therapeutic failure, based on parameters established by the Brazilian Ministry of Health and viral load counts above 5,000 copies/ $\mathrm{mL}$. A brief profile of patients failing HAART was also developed.

\section{RNA Isolation, Amplification and Genotyping}

Plasma viral RNA isolated and purified by ultra centrifugation $(120,000 \mathrm{~g}$ for $120 \mathrm{~min}$.) was submitted to a first round reverse transcription with MuLV enzyme, followed by a hot start PCR with the AmpliTaq ${ }^{\circledR}$ Gold. The DNA product that was generated corresponds to the pol fragment covering the PR and RT genes. Viral load was quantified [Nuclisens HIV-1 QT (NASBA Diagnostics, Biomerieux, Boxtel, NL)] before genotyping the samples with seven 
internal primers using the BigDyeÔ Terminator chemistry (Applied Biosystems, US). The sequencing products were visualized with an ABI 3100 Genetic Analyzer (Applied Biosystems, US).

Sequence Alignment and Phylogenetic Analysis

The generated RT and PR sequences were tested against the reference set from the Los Alamos database (hivweb.lanl.gov) for subtyping analysis. Sequences were aligned using the ClustalW algorithm and then manually corrected. Phylogenetic trees used in later analyses were built using the maximum likelihood (ML) method in PAUP*4b10.

\section{Identification of Resistance Mutations}

Sequences were submitted to the resistance test program available at Stanford University (http://hivdb.stanford.edu). This program analyses differences in amino acid sequences, including positions that contain a mixture of wild type and mutant residues, according to the consensus statement of the International AIDS Society-USA Resistance Panel[20].

\section{Statistical Analysis}

Databank and statistical analyses were done with Epi-info 2000 software. Descriptive statistics included frequency distribution, means and correlations. We used a chi-square test to examine associations of categorical variables. $P$ values $<0.05$ were considered significant.

\section{Results}

One hundred and seventeen patients failing HAART attended by RENAGENO participated in this study. The general profile of this group is summarized in Table 1 . The male gender predominated (63.2\%); however, no significant gender differences were found in the number of therapeutic regimens $(p=0.680)$, distribution of viral subtypes $(p=0.120)$ or presence of resistance mutations. The period from 1996 to 1999, especially 1997, corresponded to a major ARV therapy initiation effort (37.6\%); as expected, the period under ARV treatment was positively related with the number of therapeutic regimens used $\left(\mathrm{R}^{2}=0.868\right)$. HIV-1 subtyping of patients failing HAART revealed that subtype $B$ was the most common; it was found in 86/117 (73.5\%) samples, followed by $F$ in 7/117 (6\%) and PR/RT hybrid forms (D/B; F/B; D/F; B/F) in 7/117 (6\%) samples. In $12 \%$ of the samples, the subtype could not be determined. Subtype $\mathrm{C}$ was not detected in this group.

In 109/117 (93.1\%) samples, we identified mutations to one or more drug classes (NRTIs, NNRTIs and PIs); a large number of resistance mutations occurred in the RT gene 109/ 117 (93.1\%), in comparison with 48/117 (41.0\%) in the PR gene $(\mathrm{p}<0.001)$. We found that $6.8 \%$ of the samples had no resistance mutations. The most frequent ARV-associated resistance mutations were: M184V (60.7\%), T215Y (49.6\%), M41L (46.7\%), L210W (30.8\%) and K103N (27.3\%) in the RT gene and L90M (19.6\%), M46I (16.2\%), D30N/N88D (12.8\%) and I54V (10.2\%) in the PR gene. Table 2 shows the effects of these mutations on ARVs. Secondary mutations also predominated in PR and RT genes and could be found in all of the samples 117/117 (100\%). The most frequent were: L63P (47.0\%), L10I/F (34.2\%), V77I (29.0\%), A71V/T (26.5\%) and M36I (21.3\%) in the PR gene and I293V (62.3\%), K122E (55.7\%), I135T(55.7\%), A272P (54.1\%) and R211K (42.6\%) in the RT gene.

The number of resistance mutations tended to increase from the first (mean of 3.18 resistance mutations/sample) to the second therapeutic failure (4.39 resistance mutations/ sample) among individuals failing HAART ( $\mathrm{p}=0.079$ ); although the difference was not significant. The number of resistance mutations per sample apparently decreased or stabilized following therapeutic failures ( $p=0.875$, Table 3 ).

\section{Discussion}

In Brazil, ARVs have been shown to be effective [4]. However, these drugs also increase the possibility of developing resistance in patients with incomplete viral suppression [21,22]. In 2001, the Brazilian Ministry of Health created the National Network for HIV Genotyping (RENAGENO) to monitor HIV resistance and to examine subtype distribution over the country [23]. The genotyping test is able to detect mutations associated with phenotypic resistance of HIV to ARVs [24]; it's efficacy has been demonstrated by several clinical and molecular studies [25]. We found that the male gender predominates among patients failing HAART. This was expected, since most patients initiated therapy in the mid 90's, when the male/female rate for HIV infection was 2.5 to 3 [26]. Due to increasing heterosexual transmission during the last years, it is conceivable that this rate, now 1.5, becomes close to 1.0 [27]. The median age of the group, 36 ( \pm 4.5$)$ years, is also similar to what has been reported for the country [28]. The median quantity of ARV regimens used, $3.0( \pm 0.8)$, demonstrates the importance of the genotyping test to help guide the choice of new regimens after treatment failure $[14,18,19]$. Many scientific reports have demonstrated the superiority of treatment decisions based on genotyping tests over other protocols [29].

The distribution of subtypes that we found was similar to findings from other Brazilian studies, characterized by a B subtype domain, followed by $\mathrm{F}$ and hybrid forms, involving $\mathrm{B}, \mathrm{F}$ and D subtypes [30-32]. Although in expansion in our country, the absence of the $C$ subtype in our sample is understandable given its low prevalence in the southeast region (the origin of all the samples that we analyzed) in comparison with the southern region of the country [33].

The number of samples presenting any resistance mutation (93.1\%) was similar to what was found in other reports on patients failing HAART [34,35], although our rates were slightly elevated due to longer exposition to ARV drugs by this group of patients. This finding is of concern since resistance is a major impairment to therapeutic success [36]. NRTI drug resistance was much more frequent in comparison with resistance to other drug classes ( $\mathrm{p}<0.001)$, occurring in $93.1 \%$ of the samples. The most prevalent: M184V (60.7\%) 
Table 1. Baseline characteristics of patients failing HAART attended by RENAGENO, and corresponding viral markers (N=117).

\begin{tabular}{lccc}
\hline Patients & $\mathbf{N}$ & Viral markers & $\mathbf{N}$ \\
\hline Gender & & Viral load (median) & $46,000 \mathrm{copies} / \mathrm{mL}$ \\
$\quad$ Male & $74(63.2 \%)$ & HIV-1 subtype & $86(73.5 \%)$ \\
$\quad$ Female & $43(36.8 \%)$ & $\mathrm{B}$ & $7(6 \%)$ \\
Age range (median \pm SD) & $35-48(36 \pm 4,5)$ & $\mathrm{F}$ & $7(6 \%)$ \\
CD4 (median) & 269 cells $/ \mathrm{mm}^{3}$ & Hybrid & $14(12 \%)$ \\
No. of therapeutic regimens & & Not determined & $109(93.1 \%)$ \\
$\quad$ Range (median \pm SD) & $1-7(3 \pm 0.8)$ & Samples with resistance mutations & $117(100 \%)$ \\
Period treatment began N (\%) & & Samples with secondary mutations & $109(93.1 \%)$ \\
$\quad$ 1988-1991 & $2(1.7)$ & Presence of resistance-associated mutations & \\
1992-1995 & $6(5.1)$ & RT gene & $48(41.0 \%)$ \\
1996-1999 & $44(37.6)$ & PR gene & $8(6.48 \%)$ \\
2000-2004 & $22(18.8)$ & Wild type &
\end{tabular}

Table 2. Antiretroviral drug efficacy affected by resistance mutations found in samples from patients failing HAART (N=117).

\begin{tabular}{lcl}
\hline Resistance-associated mutations & N (\%) & Drug affected \\
\hline NRTI & & \\
M184V & $71(60.7)$ & 3TC,ABC \\
T215Y & $58(49.6)$ & ZDV, d4T \\
M41L & $43(46.7)$ & ZDV, d4T \\
L210W & $36(30.8)$ & ZDV, d4T \\
D67N & $30(25.6)$ & ZDV, d4T \\
K70R & $22(18.8)$ & ZDV, d4T, TDF \\
I74V & $10(8.5)$ & ABC, ddI \\
K219Q & $10(8.5)$ & ZDV, d4T \\
Q151M & $5(4.3)$ & ABC \\
69 insertion & $5(4.3)$ & All NRTIs \\
NNRTI & & \\
K103N & $32(27.3)$ & DLV,EFV,NVP \\
G190A & $16(13.6)$ & EFV,NVP \\
Y181C & $13(11.1)$ & DLV,EFV,NVP \\
L100I & $10(8.5)$ & EFV,NVP \\
Y188L & $9(7.7)$ & DLV,EFV,NVP \\
V108I & $3(2.6)$ & EFV,NVP \\
PI & & \\
L90M & $23(19.6)$ & NFV,SQV \\
M46I & $19(16.2)$ & IDV,RTV \\
D30N & $15(12.8)$ & NFV \\
N88D & $15(12.8)$ & ATZ, RTV \\
I54V & $12(10.2)$ & Darunavir, RTV \\
I84V & $10(8.5)$ & SQV,RTV \\
L33F & $3(2.5)$ & Tipranavir, RTV \\
I50V & $1(0.85)$ & Darunavir, RTV, Fosamprenavir, ATZ \\
\hline
\end{tabular}

*: According to The International Aids Society 2006. NRTI: nucleosidic inhibitor of reverse transcriptase; NNRTI: non- nucleosidic inhibitor of reverse transcriptase; PI: protease inhibitor; 3TC: lamivudine; ABC: Abacavir; ZDV: Zidovudine; d4T: stavudine; TDF: Tenofovir; ddI: Didanosine; DLV: Delavirdine; EFV: Efavirenz; NVP: Nevirapine; NFV: Nelfinavir; SQV: Saquinavir; RTV: Ritonavir; ATZ: Atazanavir.

and T215Y (49.6\%), responsible for resistance to 3TC/ABC and ZDV/d4T, respectively, indicates the common use of such drugs in therapeutic regimens [35]. K103N was the fifth most common mutation found (27.3\%); it accounts for groupcharacteristic cross-resistance mutations to all available
NNRTIs, indicating the growing use of such drugs as part of current HAART treatments.

PI resistance mutations (41.0\%) were less frequent than RT inhibitor mutations, as expected, due to a natural genetic PR barrier to mutations [11], and because of more recent 
Table 3. Number of resistance mutations detected in samples collected from patients failing HAART, grouped according to therapeutic scheme failure.

\begin{tabular}{ccc}
\hline No. of therapeutic scheme failures $^{\mathbf{1}}$ & No. of patients/scheme & No. of resistance mutations/sample $^{\mathbf{2}}$ mean (SD) $^{- \text {s. }}$ \\
\hline 1 & 16 & $3.18(1.75)$ \\
2 & 24 & $4.39(2.27)$ \\
3 & 34 & $4.40(2.59)$ \\
4 & 20 & $3.94(2.19)$ \\
5 & 17 & $4.0(2.72)$ \\
\hline
\end{tabular}

${ }^{1}$ Only two patients were submitted to six and seven therapeutic schemes. ${ }^{2}$ One sample per patient.

introduction of these drugs in therapy and use of alternative PIs in HAART regimens [34]. In contrast, secondary mutations, such as L63P (47\%), L10I/F (34.2\%), V77I (29\%), A71V/T (26.5\%) and M36I (21.3\%) were common in the PR gene samples. The number and pattern of such mutations was similar to that found in other studies of patients failing HAART $[37,38]$, though they may occur at similar rates in previously untreated patients [3]. These mutations configure polymorphisms that are not related to genetic resistance but are responsible for improving viral fitness [39].

The increasing number of resistance mutations from the first to the second drug failure indicates that HAART depends on the success of first regimens in order to avoid the appearance of HIV resistance variants [40]. The unexpected stabilization of resistance mutations, even after subsequent treatment failures, implies that HIV acquires a considerable number of key resistance mutations soon after treatment begins, and then stabilizes, probably avoiding further accumulation of resistance mutations that would impair subsequent viral replication [37]. This information is relevant to understanding HIV replication dynamics and sustained HAART effectiveness, although more studies that follow up patients would be necessary to support such a statement.

In conclusion, the high prevalence of HIV-1 resistance mutations and its complex genetic development during the course of ARV treatment, especially mutations against RT inhibitors, supports the necessity of sustained genotyping studies and carefully monitoring patients failing HAART.

\section{References}

1. Gallant J.E. Initial therapy of HIV infection. J Clin Virol 2002;25:317-33.

2. Werner V., Stephen B., Calvin C., et al. Prevalence of HIV drug resistance in anti-retroviral-naïve patients: a prospective study. AIDS 2001;15(5):647-50.

3. Perno C.F., Cozzi-Lepri A., Balotta C., et al. Secondary mutations in the protease region of human immunodeficiency virus and virologic failure in drug-naïve patients treated with protease inhibitor-based therapy. J Infect Dis 2001;184:983-91.

4. Marins J.R., Jamal L.F., Chen S.Y., et al. Dramatic improvement in survival among adult Brazilian AIDS patients. AIDS 2003;17(11):1675-1682.

5. Perez E.E., Rose S.L., Peyser S.L.L., Burkhart B. Human Immunodeficiency Virus Type 1 Protease Genotype Predicts Immune and Viral Responses to Combination Therapy with Protease Inhibitors (PIs) in PI-Naïve Patients. J Infect Dis 2001;183:579-88.
6. Soares M.A., Brindeiro R.M., Tanuri A. Primary HIV-1 drug resistance in Brazil. AIDS 2004;18 (suppl 3): S9-S13.

7. Ho D.D., Neumann A.U., Perelson A.S., et al. Rapid turnover of plasma virions and CD4 lymphocytes in HIV-1 infection. Nature 1995;373:123-6.

8. Brenner B., Wainberg M.A., Salomon H., Danielle R. Resistance to anti-retroviral drugs in patients with primary HIV-1 infection. Int J Anti Agents 2000;16(4):429-34.

9. Eigen M. Viral quasispecies. Sci Am 1993;269:42-9.

10. Shafer R.W., Vuitton D.A. Highly active anti-retroviral therapy (HAART) for the treatment of infection with human immunodeficiency virus type 1 . Biomed and Pharmacother 1999; 25:73-86.

11. Wu T.D., Schiffer C.A., Gonzales M.J., et al. Mutation Patterns and Structural Correlates in Human Immunodeficnecy Virus Type 1 Protease following Different Protease Inhibitor Treatments. J Virology 2003;77(8): 4836-47.

12. Ceccherini F. Identification of the minimal conserved structured of HIV-1 protease in the presence and absence of drug pressure. AIDS 2004;18:11-9.

13. Cao Y., Qin L., Zhang L., et al. Virologic and immunologic characterization of longterm survivors of human immunodeficiency virus type 1 infection. N Eng J Med 1995;332:201-8.

14. Mellors J.W., Rinaldo C.R., Gupta P., et al. Prognosis in HIV-1 infection predicted by the quantity of virus in plasma. Science 1996; 272:1167-70.

15. Hammer S.M., Türman T., Vareldziz B., et al. Antiretroviral guidelines for resource-limited settings: the WHO's public health approach. Nature Med 2002;8:649-50.

16. Ministério da Saúde. Coordenação Nacional de DST e AIDS. Projeto de Implantação de uma Rede Nacional para Genotipagem do HIV-1 (RENAGENO) em Pacientes com Falha Terapêutica aos Anti-retrovirais, 2001.

17. Baxter J.D., Mayers D.L., Wentworth D.N. A randomized study of antiretroviral management based on plasma genotypic antiretroviral resistance testing in patients failing therapy. AIDS 2000;14:F83-F93.

18. Durant J. Drug-resistance genotyping in HIV-1 therapy: the VIRADAPT randomized controlled trial. The Lancet 1999;353: 2195-9.

19. Meynard J.L. Phenotypic or genotypic resistance testing for choosing antiretroviral therapy after treatment failure: a randomized trial. AIDS 2002;16:727-36.

20. Johnson V.A., Brun-Vézinet F., Clolet B., et al. Update of the drug resistance mutations in HIV-1: fall 2006. Top HIV Med 2006;14(3):125-30.

21. Lee N., Hogg R.S., Yip B., et al. Rates of disease progression among human immunodeficiency virus-infected persons initiating multiple-drug rescue therapy. J infect Dis 2003; $188: 137-41$.

22. Little S.J., Holte S., Routy J.P., et al. Antiretroviral drugs resistance among patients recently infected with HIV. New Engl J Med 2002; 347: 385-94. 
23. Malta M., Petersen M.L., Clair S., et al. Adherence to antiretroviral therapy: a qualitative study with physicians from Rio de Janeiro, Brazil. Cad Saúde Pública 2005;21(5):1424-32.

24. Shaffer R. Genotypic testing for human immunodeficiency virus type 1 drug resistance Clinical Microbiology Reviews 2002;15:247-77.

25. D’Aquila R.T. Incorporating Anti-retroviral Resistance Testing Into Clinical Practice. Medical Education Collaborative 2002.

26. Chequer P. Apresentação do boletim de Aids. In: Coordenação Nacional de DST e Aids (ed.). Boletim Epidemiológico de Aids 2004. Brasília: Ministério da Saúde; 2004.

27. Brito A.M., Castilho E.A., Szwarcwald C.L. Aids e infecção pelo HIV no Brasil: uma epidemia multifacetada. Rev Soc Bras Med Trop 2000;34:207-17.

28. Szwarcwald C.L., Bastos F.I., Andrade C.L.T., et al. Aids: o mapa ecológico do Brasil, 1982-1994. In: Coordenação Nacional de DST e Aids (ed.). A epidemia da Aids no Brasil: situação e tendências. Brasília: Ministério da Saúde; 1997. p. 27-44.

29. Morgado M.G., Guimaraes M.L., Gripp C.B., et al. Molecular epidemiology of HIV-1 in Brazil: high prevalence of HIV-1 subtype B and identification of an HIV-1 subtype D infection in the city of Rio de Janeiro, Brazil. J Acquir Immune Defic Syndr 1998; 18(5):488-94.

30. Tupinambás U., Ribeiro F.A., Aleixo A., Greco D. Treatment switch guided by HIV-1 genotyping in Brazil. Braz J Infect Dis 2006;10(2):82-8.

31. Da Costa S.M., Schechter M., Shindo N., et al. Sequence and phylogenetic analysis of glycoprotein 120 of an HIV type 1 variant (GWGR) prevalent in Brazil. AIDS Res Hum Retroviruses 1998;11(9):1143-5.

32. Sabino E.C., Diaz R.S., Brigido L.F., et al. Distribution of HIV-1 subtypes seen in an AIDS clinic in Sao Paulo City, Brazil. AIDS 1996;10(13):1579- 84 .
33. Brindeiro R.M., Diaz R.S., Sabino E.C., et al. Brazilian Network for HIV Drug Resistance Surveillance (HIV-BResNet): a survey of chronically infected individuals. AIDS 2003;17:1063-9.

34. Couto-Fernandez J.C., Silva-de-Jesus C., Rachid M., et al. Human immunodeficiency virus type 1 (HIV-1) genotyping in Rio de Janeiro, Brazil: assessing subtype and drugresistance associated mutations in HIV-1 infected individuals failing highly active antiretroviral therapy. Mem Inst Oswaldo Cruz 2005; 100(1): 73-8.

35. Tanuri A., Caridea E., Dantas M.C., et al. Prevalence of mutations related to HIV-1 antiretroviral resistance in Brazilian patients failing HAART. J Clin Virol 2002;(25) 39-45.

36. Castilho L.T., Herrera-Martínez G., León-Bratti M., et al. Study of antiretroviral mutants in HIV patients with treatment failures and the effect of risk factors in the virological failures. Rev Inst Med trop S Paulo 2005; 47(6): 327-31.

37. Kristiansen T.B., Pedersen A.G., Eugen-Olsen J., et al. Genetic evolution of HIV in patients on stable HAART regimen despite insufficient viral suppression. Scandinavian J Infect Dis 2005;37: 890-1.

38. Harrigan P.R., Hogg R.S., Dong W.W.Y., et al. Predictors of HIV drug-resistance mutations in a large antiretroviral-naïve cohort initiating triple antiretroviral therapy. $\mathrm{J}$ Infect Dis 2004;191:339-47.

39. Leal E.S., Holmes E.C., Zanotto P.M. Distinct patterns of natural selection in the reverse transcriptase gene of HIV-1 in the presence and absence of antiretroviral therapy. Virology 2004;325:181-91.

40. Louie M., Markowitz M. Goals and milestones during treatment of HIV-1 infection with anti-retroviral therapy: a pathogenesis-based perspective. Antiviral Research 2002;55:5-15. 